\title{
MODELING SPACE CHARGE IN BEAMS FOR HEAVY-ION FUSION*
}

\author{
W. M. Sharp \\ Lawrence Livermore National Laboratory L-440, Livermore, CA 94550, USA
}

\begin{abstract}
A new analytic model is presented which accurately estimates the radially averaged axial component of the space-charge field of an axisymmetric heavy-ion beam in a cylindrical beam pipe. The model recovers details of the field near the beam ends that are overlooked by simpler models, and the results compare well to exact solutions of Poisson's equation. Field values are shown for several simple beam profiles and are compared with values obtained from simpler models.
\end{abstract}

\section{INTRODUCTION}

Longitudinal confinement of space-charge-dominated beams in induction accelerators requires detailed knowledge of the beam space-charge field. Unlike radio-frequency accelerators, the accelerating fields of induction accelerators provide no longitudinal focusing, so time-varying electric fields must be added to the acceleration field in at least some induction modules to balance the space-charge force. For the ion beams considered for heavy-ion fusion (HIF), which are typically meters long and only a few centimeters in radius, these longitudinalcontrol fields, referred to here as "ears," are highly non-linear and must be calculated from the measured beam quantities like current and radius.

In HIF experiments [1] and some analytic work [2], the beam space-charge field has been calculated from a simple onedimensional model. By assuming axisymmetry and a uniform charge density $\rho$, it can be shown that neglecting the axial derivative in Poisson's equation leads to the simple result, in SI units, that

$$
\left\langle E_{z}(z)\right\rangle \approx-\frac{1}{4 \pi \epsilon_{0}}\left[\ln \left(\frac{R^{2}}{a^{2}}\right)\right] \frac{\partial \lambda}{\partial z} .
$$

Here, $\lambda=\pi \rho a^{2}$ is the beam line-charge density, $z$ is axial distance in the beam frame, and the angle brackets denote averaging over the beam cross section. The logarithmic factor in Eq. (1) is call the "geometry factor" or "g-factor," and $a$ and $R$ in the term denote the radii of the beam and the beam pipe respectively. A slightly more sophisticated treatment, including the possible axial variation in $a$ gives

$$
\left\langle E_{z}(z)\right\rangle \approx-\frac{1}{4 \pi \epsilon_{0}}\left\{\left[\frac{1}{2}+\ln \left(\frac{R^{2}}{a^{2}}\right)\right] \frac{\partial \lambda}{\partial z}-\frac{\lambda}{a} \frac{\partial a}{\partial z}\right\}
$$

These simple expressions are not expected to be valid at the beam ends because neglecting the axial derivative in Poisson's equation is clearly invalid there. The failure of qs. (1) and (2)

\footnotetext{
* The research was performed under the auspices of the U. S. Department of Energy by Lawrence Livermore National Laboratory under Contract No. W7405-ENG-48.
}

is evident, for example, for a beam with a uniform charge density. For this case, $a$ vanishes at the beam ends, and both $\left\langle E_{z}\right\rangle$ expressions unphysically become singular there.

In this paper, a Green's function is used to derive a more general expression for the radially averaged axial space-charge field $\left\langle E_{z}\right\rangle$ of a nonrelativistic ion beam centered in a perfectly conducting cylindrical pipe. The expression is specialized to beams, like those in induction accelerators, that are much longer than their radius, and a closed-form approximation to $\left\langle E_{z}\right\rangle$ is obtained for the class of beams with $a / R \gtrsim 0.05$ at all points. This calculation is done in the beam frame, but since HIF beams are nonrelativistic, $\left\langle E_{z}\right\rangle$ is effectively the same in the laboratory frame. The importance of beam-radius variation is illustrated by plotting the space-charge field for several beam profiles, and results of the new model are compared with predictions of the simpler g-factor models.

\section{DERIVATION}

A general expression for $\left\langle E_{z}\right\rangle$ is derived from a Green's function equivalent to that in Ref. [3]. The Green's function $G$ for the potential of a ring of charge with unit magnitude centered in a perfectly conducting pipe of radius $R$ is obtained from Poisson's equation, given in SI units for this case by

$$
\nabla^{2} G\left(r, z ; r^{\prime}, z^{\prime}\right)=\frac{1}{\epsilon_{0} r} \delta\left(r-r^{\prime}\right) \delta\left(z-z^{\prime}\right),
$$

where the primed coordinates denote the source location, and unprimed coordinates are field points. A straightforward solution gives

$$
\begin{gathered}
G\left(r, z ; r^{\prime}, z^{\prime}\right) \\
=\frac{1}{\epsilon_{0} R} \sum_{n=1}^{\infty} \frac{J_{0}\left(\frac{\alpha_{n}}{R} r\right) J_{1}\left(\frac{\alpha_{n}}{R} r^{\prime}\right)}{\alpha_{n} J_{1}^{2}\left(\alpha_{n}\right)} \exp \left(-\frac{\alpha_{n}}{R}\left|z-z^{\prime}\right|\right),
\end{gathered}
$$

where $J_{0}$ and $J_{1}$ are Bessel functions of the first kind, and $\alpha_{n}$ denotes the $n$th zero of $J_{0}$. The potential $\phi$ for any axisymmetric charge distribution with density $\rho(r, z)$ is then found by integrating $G$ over all $r^{\prime}$ and $z^{\prime}$, and the corresponding axial space-charge field is given by $E_{z}(r, z)=-\partial \phi(r, z) / \partial z$. When $\rho$ is assumed to be independent of $r$ within some radius $a(z)$, then the $E_{z}$ expression is trivially averaged over $r$, giving

$$
\begin{gathered}
\left\langle E_{z}(z)\right\rangle=\frac{1}{\pi \epsilon_{0} R} \sum_{n=1}^{\infty} \frac{\left(\frac{2}{A_{n} a}\right) J_{1}\left(A_{n} a\right)}{\alpha_{n} J_{1}^{2}\left(\alpha_{n}\right)} \\
{\left[\int_{-L_{b} / 2}^{L_{b} / 2} d z^{\prime} \operatorname{sgn}\left(z-z^{\prime}\right) \frac{\lambda^{\prime}}{a^{\prime}} J_{1}\left(A_{n} a^{\prime}\right) \exp \left(-A_{n}\left|z-z^{\prime}\right|\right)\right] .}
\end{gathered}
$$

Here, the notation $A_{n} \equiv \alpha_{n} / R$ has been introduced, and $z$ has been assumed to be zero at the beam midpoint, so that the ends of a beam of length $L_{b}$ are at $\pm L_{b} / 2$. 
The integral in Eq. (5) cannot in general be evaluated in closed form. However, for typical beams from induction accelerators, the axial scale lengths of $a$ and $\lambda$ are much longer than the exponential scale length $A_{n}^{-1}$ for all $n$. This short exponential scale length allows us to expand the integrand linearly about the $z^{\prime}$ value where the integrand magnitude is maximum. Equating the derivative of the integrand with respect to $z^{\prime}$ to zero gives a transcendental equation for this integrand extremum. Rather than solve this equation numerically, we simplify the equation by assuming that the Bessel-function arguments are small, as is appropriate when $A_{n} a^{\prime} \lesssim 1$. The resulting equation for the location of the integrand peak is

$$
1-\operatorname{sgn}\left(z-z^{\prime}\right) A_{n} \zeta^{\prime} \approx 0,
$$

where $\zeta(z)=\left(L_{b} / 2\right)-|z|$ is the axial distance from the nearest beam end. Examination of Eq. (7) shows that the integrand peak is

$$
\zeta^{\prime} \approx \max \left(\zeta, A_{n}^{-1}\right) \equiv \zeta_{n} .
$$

The significance of Eq. (7) is that the integrand is expanded about the exponential maximum except very near the ends. The approximation leading to Eq. (7) proves to be excellent in cases where $a\left( \pm L_{b} / 2\right) \ll R$, and it still is usable for larger beam-end radii because $a$ varies slowly near the beam ends, so errors in $\zeta_{n}$ have little consequence.

After linear expansion of the integrand about $\zeta_{n}$, the integral in Eq. (5) is evaluated in a straightforward manner and, after some algebra, gives the following Bessel-series expression for $\left\langle E_{z}\right\rangle$ :

$$
\begin{gathered}
\left\langle E_{z}(z)\right\rangle=\frac{1}{\pi \epsilon_{0}} \sum_{n=1}^{\infty} \frac{\left(\frac{2}{A_{n} a}\right) J_{1}\left(A_{n} a\right)}{\alpha_{n}^{2} J_{1}^{2}\left(\alpha_{n}\right)} \\
\left\{\operatorname{sgn}(z) \frac{\lambda_{n}}{a_{n}} J_{1}\left(A_{n} a_{n}\right) \exp \left(-A_{n} \zeta\right)\right. \\
\left.-2\left[\frac{1}{A_{n} a} \frac{\partial \lambda}{\partial z} J_{1}\left(A_{n} a\right)-\frac{\lambda}{a} \frac{\partial a}{\partial z} J_{2}\left(A_{n} a\right)\right]_{\zeta_{n}} f_{n}(z)\right\},
\end{gathered}
$$

where $a_{n}$ and $\lambda_{n}$ are values at $\zeta_{n}$, and

$$
f_{n}(z) \equiv 1-\frac{1}{2}\left[1+\max \left(1, A_{n} \zeta\right)\right] \exp \left(-A_{n} \zeta\right) .
$$

Here, the fact that $A_{n} L_{b} \gg 1$ has been used to discard exponentially small contributions from the farther of the two beam ends. This expression is found to be in excellent agreement with the exact expression Eq. (5) for every case examined.

Eq. (9) is an important result of this paper, but the summation in general requires laborious numerical evaluation. In the following section, however, it is shown that the expression may be approximately evaluated for beams with sufficiently large radii at the beam ends.

\section{SPECIAL CASES}

\section{A. Beam Profiles}

The radius $a$ of the axisymmetric beam and the line-charge density $\lambda$ in Eq. (9) are in general independent quantities related by the beam transverse emittance and the accelerator lattice. In this work, $\lambda$ is taken to be any non-negative function that vanishes smoothly at the beam ends. For the equilibrium axisymmetric beams considered here, $a, \lambda$, and the normalized edge emittance $\epsilon_{N}$ are related approximately by the steady-state envelope equation

$$
-\frac{\sigma_{0}^{2}}{4 L^{2}} a+\frac{\kappa}{a}+\frac{\beta^{2} \epsilon_{N}^{2}}{a^{3}} \approx 0 .
$$

Here, $\sigma_{0}$ is the phase advance per lattice period $2 L$ in the absence of space-charge effects, and

$$
\kappa=\frac{1}{4 \pi \epsilon_{0}} \frac{2 e \lambda}{\beta^{2} M c^{2}} \equiv K \frac{\lambda}{\beta^{2}}
$$

is the beam perveance, with $\beta$ being the beam axial velocity scaled by $c$. Four cases are studied here:

(1) Uniform radius. Here, $\epsilon_{N}$ is obtained directly from Eq. (10) and increases toward the beam ends to balance the decreasing transverse space-charge force.

(2) Uniform normalized emittance. For this case, Eq. (10) is solved trivially for $a^{2}$, giving

$$
a^{2} \approx \frac{2 L^{2}}{\sigma_{0}^{2}}\left[K \frac{\lambda}{\beta^{2}}+\left(K^{2} \frac{\lambda^{2}}{\beta^{4}}+\frac{\beta^{2} \epsilon_{0}^{2} \sigma_{0}^{2}}{L^{2}}\right)^{1 / 2}\right] .
$$

(3) Uniform "transverse temperature". Even though the envelope equation Eq. (10) is derived under the assumption that the beam is cold in the transverse plane, the transverse temperature of a bean is in general proportional to $T \equiv \epsilon_{N}^{2} / a^{2}$. If this temperature-like quantity is treated as uniform along a beam, Eq. (10) gives

$$
a^{2} \approx \frac{4 L^{2}}{\sigma_{0}^{2}}\left(K \frac{\lambda}{\beta^{2}}+T\right) .
$$

(4) Uniform charge density. If the charge density $\rho$ is assumed uniform along the beam, then

$$
a^{2}=\frac{\lambda}{\pi \rho},
$$

and the normalized emittance $\epsilon_{N}$ from q. (10) vanishes at the beam ends along with $\lambda$ and $a$.

Although these simple profiles are unlikely to match that in an experimental beam, they illustrate the sensitivity of the spacecharge field to the beam radial variation. Fig. 1 shows field values calculated for beams with identical parameters and linecharge profiles, but differing radial profiles. The parameters are those of a small recirculating induction accelerator being built at the Lawrence Livermore National Laboratory [4], except that the midsection of the beam has been shortened to highlight field changes near the ends. As expected, one finds that the peak space-charge field increases for profiles that have smaller end radii. It is also evident that $\left\langle E_{z}\right\rangle$ for the uniform-density is qualitatively different from the others. For the cases with a finite beam-end radius, the field magnitude is seen to drop significantly in a narrow region at the beam end. In this region, which has a characteristic length of $R / \alpha_{1}$, the absence of charge outside the beam reduces the axial field, and at the endpoints, the field is reduced by approximately half. In contrast, $\left\langle E_{z}\right\rangle$ 


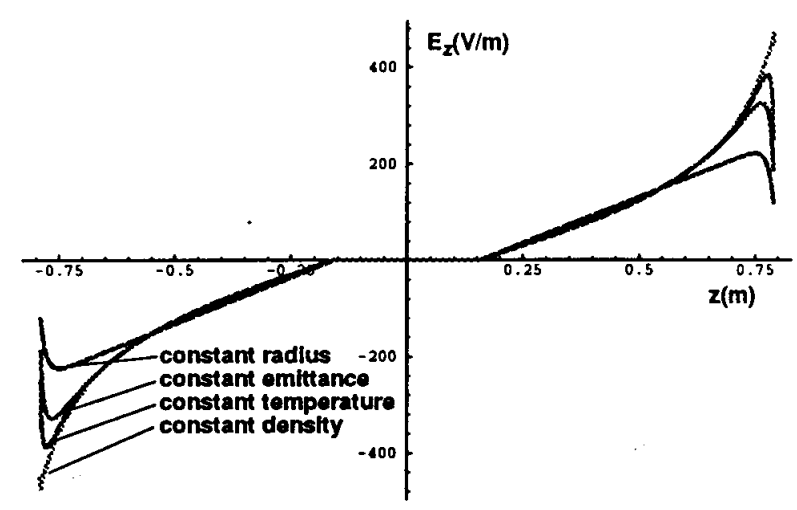

Figure 1. Radially averaged space-charge field for beams with various radial profiles but the same line- charge density.

for the uniform-density case varies monotonically near the end due to the rapidly decreasing radius. Another distinction of the uniform-density case is that about 250 terms are required for convergence of the Bessel series in Eq. (8), whereas the other cases require between 20 and 40 terms. This difference arises because the beam radial profile is poorly fit by a Bessel series when $a / R$ is small, and many terms are needed for an adequate representation.

\section{B. Analytic Approximations}

Since the series Eq. (8) converges rapidly when $a / R \gtrsim 0.05$ for all $z$, it is sensible to approximate the $\left\langle E_{z}\right\rangle$ expression by setting $n=1$ in $f_{n}$ and in the exponential factor. Also, since $a$ varies only slightly between the ends and $\zeta_{1}$ for such beams, leading Bessel factor and the derivatives of $a$ and $\lambda$ can all be evaluated at $\zeta_{1}$ with negligible error. These approximations leave two Bessel series that, remarkably, can be exactly summed. Expressed generally, these Bessel sums have been verified numerically over the range $1 \geq x>0$ :

$$
\begin{aligned}
& 4 \sum_{n=1}^{\infty} \frac{\left(\frac{2}{\alpha_{n} x}\right)^{2} J_{1}^{2}\left(\alpha_{n} x\right)}{\alpha_{n}^{2} J_{1}^{2}\left(\alpha_{n}\right)}=\frac{1}{2}+\ln \left(\frac{1}{x^{2}}\right) \\
& 8 \sum_{n=1}^{\infty} \frac{\left(\frac{2}{\alpha_{n} x}\right) J_{1}\left(\alpha_{n} x\right) J_{2}\left(\alpha_{n} x\right)}{\alpha_{n}^{2} J_{1}^{2}\left(\alpha_{n}\right)}=1 .
\end{aligned}
$$

Outside the specified range, these sums either fail to converge or give other values. Substituting Eq. (15) into the approximate form of Eq. (8) leads to the expression

$$
\begin{gathered}
\left\langle E_{z}(z)\right\rangle=-\frac{1}{4 \pi \epsilon_{0}}\left\{[ \frac { 1 } { 2 } + \operatorname { l n } ( \frac { r ^ { 2 } } { a _ { 1 } ^ { 2 } } ) ] \left[\left.f_{1}(z) \frac{\partial \lambda}{\partial z}\right|_{\zeta_{1}}\right.\right. \\
\left.-\operatorname{sgn}(z) \frac{A_{1} \lambda_{1}}{2} \exp \left(-A_{n} \zeta\right)\right]-f_{1}(z)\left[\frac{\lambda}{a} \frac{\partial a}{\partial z}\right]_{\zeta_{1}} .
\end{gathered}
$$

This expression is a numerically tractable generalization of Eq. (2) and is a very good approximation to the Bessel-series expression Eq. (8) for most experimental beams. As expected, it gives an inaccurate but non-singular result for uniform-density beams.

Sufficiently far from the beam ends, the exponentials in Eq. (8) vanish, and $\zeta_{n}=\zeta$. In this case, the Bessel sums in

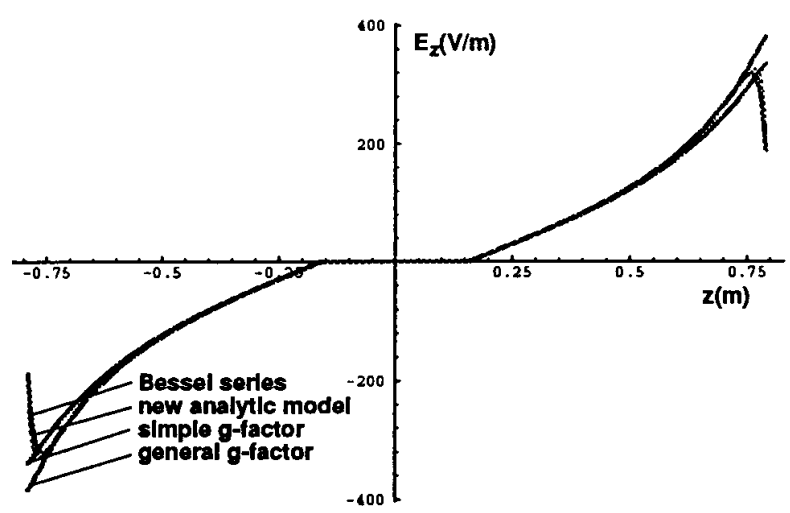

Figure 2. Radially averaged space-charge field for a constant emittance beam calculated using various approximations

Eq. (15) can be used to evaluate Eq. (8) without further approximation, and the result exactly recovers the general g-factor expression Eq. (2). It follows that Eq. (2) gives $\left\langle E_{z}\right\rangle$ accurately in the interior of any beam in which $\lambda$ and $a$ vary on length scales that are long compared with $R / \alpha_{1}$. The expression only fails within a region a few times $R / \alpha_{1}$ in length at each end.

The various approximate expressions for $\left\langle E_{z}\right\rangle$ are compared in Fig. 2 for a beam with a uniform normalized emittance and the same lattice and beam parameters as the beams shown in Fig. 1. The Bessel-series approximation has been compared with the exact integral expression Eq. (5) at selected points along the beam and is found to agree within $1 \%$ everywhere. The new analytic expression Eq. (16) deviates from the Bessel-series result by a few percent near the peak magnitude of $\left\langle E_{z}\right\rangle$, but it nonetheless reproduces the main features of the more exact expression. Both curves overlay the general g-factor model away from the ends, as expected. The curve generated from the simple g-factor expression Eq. (1) deviates from the other approximations in the beam interior, underlining the fact that variation of the beam radius cannot in general be ignored.

\section{References}

[1] T. J. Fessenden, D. L. Judd, D. Keefe. C. Kim, L. J. Laslett, L. Smith, and A. I. Warwick, AIP Conference Proceedings 152 from the 1985 Heavy-Ion Fusion Symposium, Washington, D. C., p. 145.

[2] E. P. Lee, Proceedings of the 1981 Linear Accelerator Conference, Los Alamos Report LA-9234-C, p. 263.

[3] C. K. Allen, N. Brown, and M. Reiser, Part. Accel. 45, 149 (1994).

[4] A.Friedman, J. J. Barnard, M. D. Cable, D. A. Callahan, F. J. Deadrick, S. Elon, T. J. Fessenden, D. P. Grote, D. L. Judd, H. C. Kirbie, D. B. Longinotti, S. M. Lund, L. A. Natrass, M. .B .Nelson, M. A. Newton, T. C. Sangster, W. M. Sharp, and S. S. Yu, "Progress Toward a Prototype Recirculating Induction Accelerator for Heavy-Ion Fusion," in these proceedings. 